Chaisson: We hope the first study will show that moxifloxacin speeds up the sterilization time in sputum samples from TB patients, and then we hope to show that a regimen given for four months is as effective as one given for six months. We will also continue to look at the other drugs in the pipeline and make adjustments to the regimens as the trials proceed.

JCI: What do you consider to be your greatest scientific achievement?

Chaisson: I have made some contributions that have been worthwhile, I hope. Early on, I was able to show the importance of the interaction of TB and HIV in San Francisco. Over the past few years we have built on this work and explored the public health dimensions of TB and HIV, developing new models of TB control in the era of HIV. I was able to show that supervised treatment for TB improved survival and led to reduced incidence in Baltimore. And back when I was largely focused on HIV, my colleague Richard Moore and I pioneered the use of HIV clinical cohorts, which has led to numerous important observations on the natural history and treatment outcomes of HIV infection and played a critical role in developing treatment guidelines globally.

JCI: What about your greatest accomplishment in life outside of science?

Chaisson: My greatest non-work-related accomplishment is having a wonderful family, for which I can only take a small amount of credit. I was lucky enough to marry a wonderful woman, Judy Harding, and we have two teenage daughters who give us nothing but joy.

JCI: In addition to the moxifloxacin study, you also direct the Consortium to Respond Effectively to the AIDS/TB Epidemic (CREATE), an international effort to treat and control the spread of tuberculosis in countries hit hardest by the two epidemics. What has been your most interesting experience in that realm?

Chaisson: The work I am doing on CREATE has drawn the attention and support of some very important people, not the least of whom are Nelson Mandela and Desmond Tutu. Mr. Mandela attended the launch of CREATE in Bangkok last year, and gave one of the most important TB advocacy speeches in history. Archbishop Tutu attended our last investigators' meeting in Cape Town in September and gave the most moving speech I have heard in years.

\section{Stacie Bloom}

\title{
Taking the direct route to make open access even easier
}

F entific nonprofit publishers in the US have offered the NIH direct links to their journals to make research access even easier for the public, and at no cost to boot.

Their proposal, sent to NIH director Elias Zerhouni on October 25, laid out a plan for the NIH to provide access to their journal websites using links from abstracts that are already in place and indexed on Medline. This system would bring the NIH a step closer to their known goal of complete public access to all NIH-funded research. Currently, the $\mathrm{NIH}$ requests that all grantees submit their manuscripts as soon as they have been accepted for publication, before they have benefited from production changes such as copy editing and layout.

A major concern has been the potential of confusion if more than one version of the manuscript is made available, which could lead to errors. The new proposal would eliminate this worry - the publishers are offering the finalized manuscripts to the NIH at no charge so that only the published version of the paper would be available, keeping the scientific record intact while maintaining copyright protections. This arrangement would also eliminate the need to create a taxpayerfunded infrastructure that would support a web database to publish and house the penultimate versions of the papers and will permit seamless linkage to more than 1 million scientific articles, a number that will increase by approximately 15,000 each month.

"Overnight and at no cost to taxpayers, this proposal will make it easy for the public to access vast amounts of the most accurate scientific and medical information available," said Chris Lynch, vice president of publishing for the Massachusetts Medical Society, in a press release. "What we are proposing is for the NIH to become the public's doorway into the universe of research that non-profit publishers already provide to the public everyday."

"This is simply another step in fostering collaboration among the scientific communities and the public," said Lenne P. Miller of The Endocrine Society. "We look forward to hearing back from Director Zerhouni and to meeting with him to move forward on an exciting partnership that would deliver enormous public benefits for generations to come."

\section{SB}

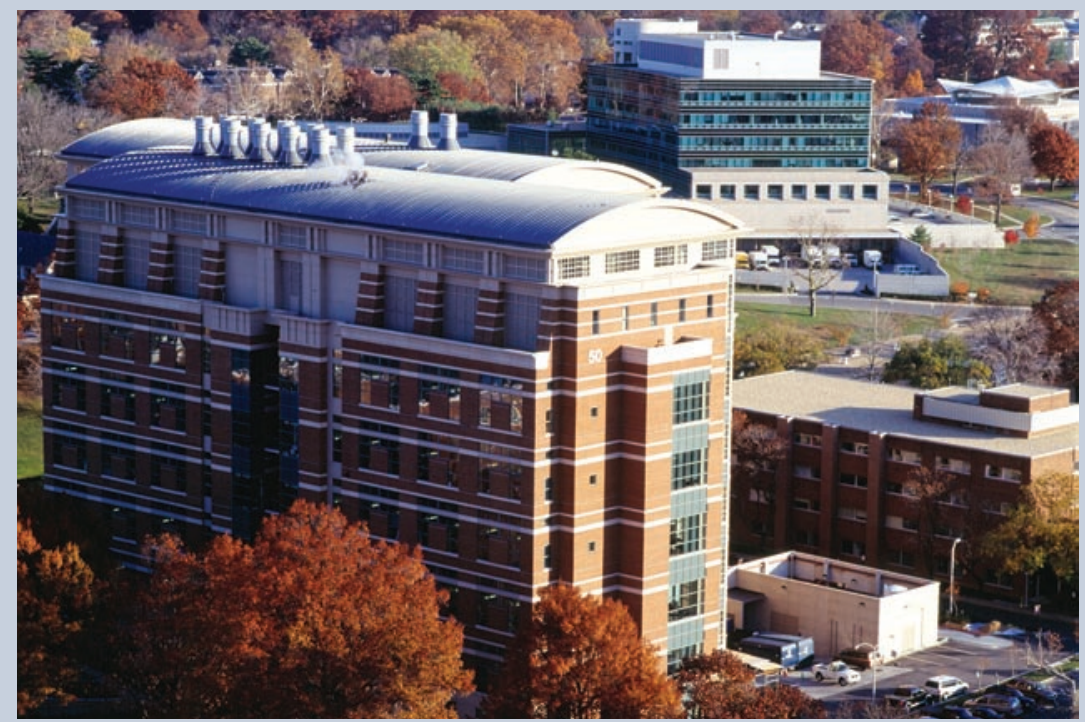

To foster easy public access, a leading group of nonprofit publishers have offered the $\mathrm{NIH}$ direct links to their journals. 